\title{
Heartbeat: racial and ethnic healthcare disparities in cardiovascular care
}

Racial and ethnic healthcare disparities in cardiovascular care have been magnified during the COVID-19 pandemic. In this issue of Heart, Rashid and colleagues ${ }^{1}$ compared admission rates, treatment and mortality of black, Asian and minority ethnic (BAME) patients with acute myocardial infarction (AMI) in England in 2020 compared with the 3 previous years. Compared with white patients, a higher proportion of BAME patients were hospitalised with AMI during the pandemic (figure 1). However, in those with AMI, BAME patients less often underwent coronary angiography $(86.1 \%$ vs $90.0 \%$, $\mathrm{p}<0.001$ ), had a longer median delay to reperfusion (4.1 hours vs 3.7 hours, $\mathrm{p}<0.001$ ) and a higher in-hospital (OR $1.68,95 \%$ CI 1.27 to 2.28 ) and 7 -day mortality (OR $1.8195 \%$ CI 1.31 to 2.19 ).

In the accompanying editorial, Cader $e t$ $a l^{2}$ point out that although differences in patient acuity might account for some of these disparities, the delays in treatment, lower rates of angiography and less adherence to guideline-mandated care 'fully qualify as evident healthcare inequalities and reinforce disconcerting disparities in cardiovascular care affecting BAME populations.' The authors go on to propose specific achievable approaches to address these inequities both at the public policy and individual physician or health-care provider level (table 1).

Current recommendations about stroke risk in patients with rheumatic heart disease (RHD) are based on very limited evidence. Additional data is provided in this issue of Heart by Vasconcelos and colleagues $^{3}$ with a prospective study of 511 consecutive patients (85\% women) with RHD (80\% mitral valve) followed for a median 3.9 years. All patients with atrial fibrillation (AF) at baseline or a prior stroke $(18 \%)$ were on warfarin anticoagulation. Even so, a new ischaemic stroke occurred in $5.2 \%$, corresponding to an incidence of 1.47 per 100 patient-years (figure 2). Taking into consideration death

Division of Cardiology, University of Washington, Seattle, USA

Correspondence to Professor Catherine M Otto, Division of Cardiology, University of Washington, Seattle, WA 98115, USA; cmotto@uw.edu

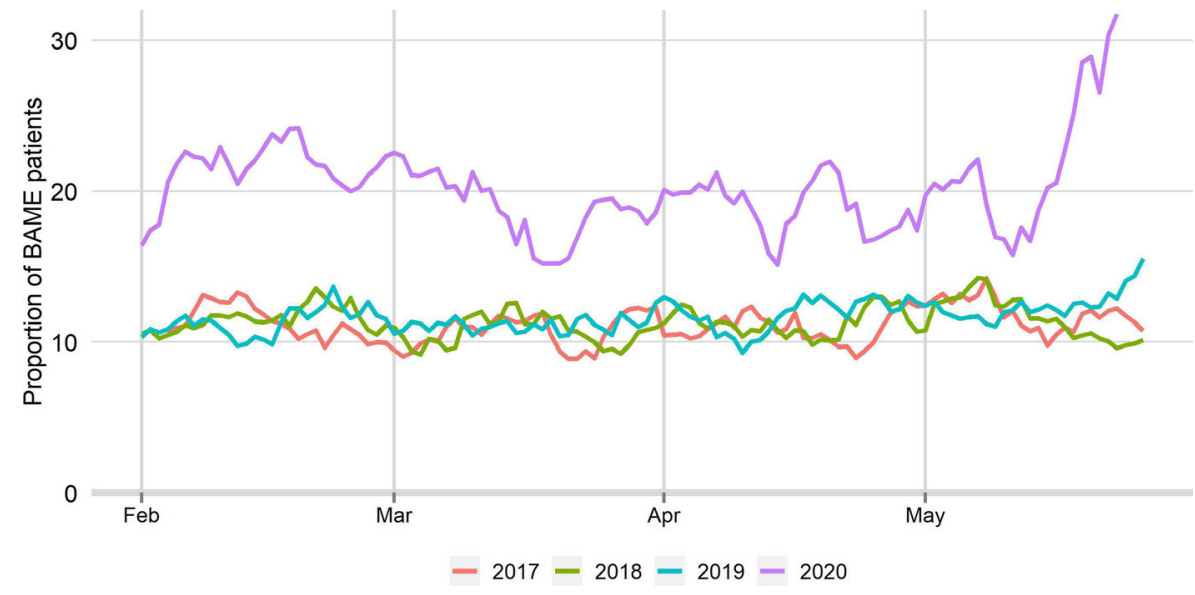

Figure 1 Time series plot of daily proportions of BAME patients hospitalised with diagnosis of AMI from 1 January 2017 to 27 May 2020 stratified according to the year of admission. Lines represent a 14-day simple moving average (indicating the mean number of daily admissions for that day and the preceding 13 days) up to and including 22 March 2020. For data from 23 March 2020, a 7-day moving average (indicating the mean number of admissions for that day and the preceding 6 days) up to and including 27 May 2020, adjusted for seasonality was plotted. AMI, acute myocardial infarction; BAME, black, Asian and minority ethnic.

as a competing risk, predictors of incident stroke were age, AF and prior stroke.

Karthikeyan ${ }^{4}$ argues that that risk stratification for stroke risk is needed in patients with RHD and AF, just as in patients with non-valvular AF. As he points out: 'AF in patients with RHD is largely restricted to patients who have severe mitral valve disease (particularly mitral stenosis) and left atrial hypertension and dilatation, all of which may contribute to the risk of stroke. Therefore, AF in RHD may be more a marker of severe disease and a less robust predictor of stroke (after adjustment for the other metrics of disease severity).' 
Atrial fibrillation - No $-\cdot$ Yes

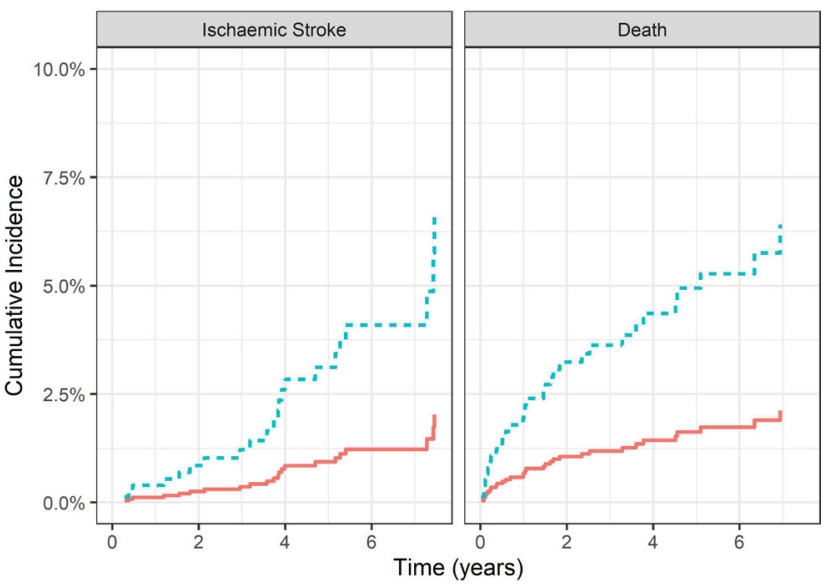

Figure 2 Incidence of ischaemic stroke and death without stroke according to the presence of AF at baseline using the Kaplan-Meier survival. The incidence of stroke stratified by the presence of AF is displayed on the left panel, and the incidence of death according to the presence of AF is shown on the right panel. The HR of AF for predicting stroke was 4.148 (95\% CI 1.896 to 9.078), whereas for predicting death was 3.168 (95\% Cl 1.575 to 6.375$)$. AF, atrial fibrillation.

The complex interaction of AF with other risk factors and competing clinical outcomes was also seen in a study from Japan $^{5}$ of 1159 patients with AF but without pre-existing heart failure (HF). The mean age was 72 years, the median $\mathrm{CHA}_{2} \mathrm{DS}_{2}$-VASc score was 3 , and $56 \%$ were on oral anticoagulation therapy. In

this cohort, higher N-terminal pro-B-type natriuretic peptide (NT-proBNP) levels were associated with a higher incidence of stroke/systemic embolism, all-cause death and HF hospitalisation during a median follow-up period of 5.0 years (figure 3 ).

In the accompanying editorial, Guasch $^{6}$ concisely summarises the
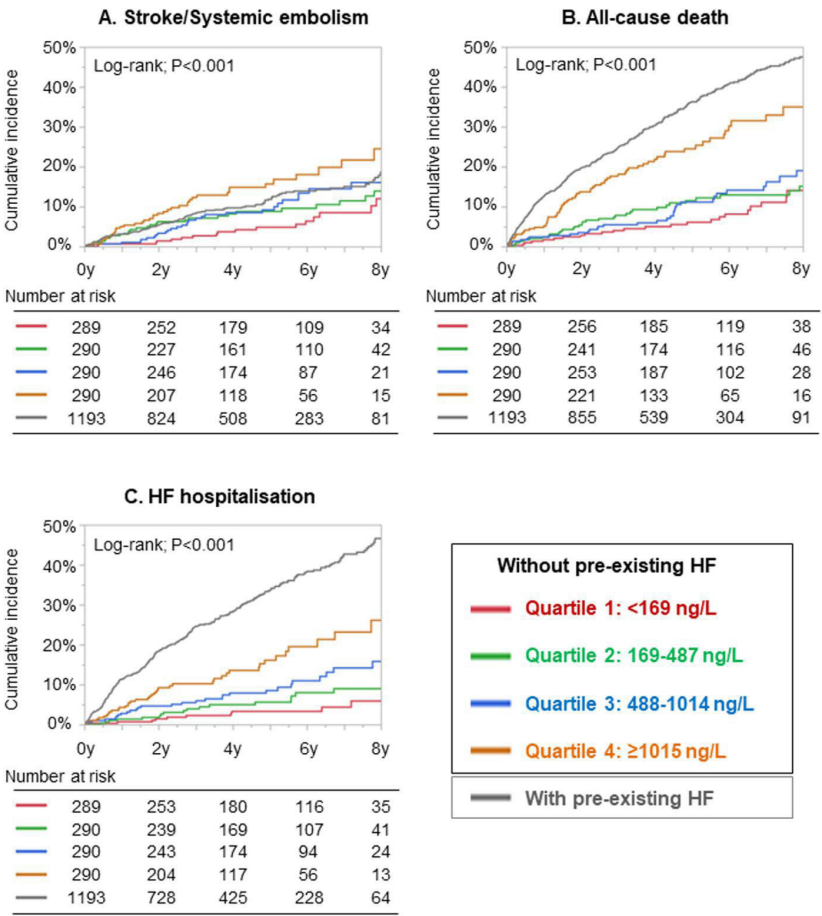

Figure 3 Kaplan-Meier curves for the incidences of clinical outcomes according to the quartiles of N-terminal pro B-type natriuretic peptide levels among patients without pre-existing HF in reference to those with pre-existing HF. (A) Stroke/systemic embolism: Q2 versus Q1: $p=0.12, Q 3$ versus $Q 1: p=0.046, Q 4$ versus $Q 1: p<0.001$. (B) All-cause death: $Q 2$ versus $Q 1: p=0.21, Q 3$ versus $\mathrm{Q} 1: \mathrm{p}=0.14, \mathrm{Q} 4$ versus $\mathrm{Q1}: \mathrm{p}<0.001$. (C) HF hospitalisation: $\mathrm{Q} 2$ versus $\mathrm{Q} 1: \mathrm{p}=0.15, \mathrm{Q} 3$ versus $\mathrm{Q} 1$ : $p=0.002$, Q4 versus $Q 1: p<0.001$. HF, heart failure; $Q$, quartile. complexities of the association between $\mathrm{AF}$ and adverse cardiovascular outcomes and the challenges in addressing these issues at the patient and population level. 'When making a diagnosis of atrial fibrillation (AF), we are all of a sudden flagging our patient with an increased risk of stroke, heart failure and death.' $\mathrm{He}$ points out that anticoagulation treatment reduces stroke risk but not the risk of heart failure hospitalisation and death. He proposes an approach that includes risk factor management, a rhythm control strategy in high-risk patients, and consideration of catheter AF ablation when systolic heart failure is present. The question raised by the study of Hamatani and colleagues ${ }^{5}$ is whether NT-proBNP levels might allow improved risk stratification of AF patients or guide earlier intervention in AF patients with elevated NT-proBNP levels.

The Education in Heart article ${ }^{7}$ in this issue details the use of intracoronary imaging to guide percutaneous coronary interventions. Either intravascular ultrasound (IVUS) or optical coherence tomography (OCT) can be used safely in most patients with the choice between IVUS and OCT determined by the utility of each approach in improving patient outcomes, not just angiographic guidance. For example, IVUS is preferred over OCT in patients with renal dysfunction to reduce contrast volume. On the other hand, OCT is preferred for ruptured plaque and thrombus due to superior resolution and detection of erosions, which is associated with a reduction in all-cause and cardiac mortality. Interested readers will find additional details and images in this excellent article.

The Cardiology-in-Focus article ${ }^{8}$ addresses the ethical issues in personal responsibility for cardiac health, something we all encounter in caring for patients with cardiovascular disease. The article focuses on moral responsibility defined as: 'Moral responsibility for that

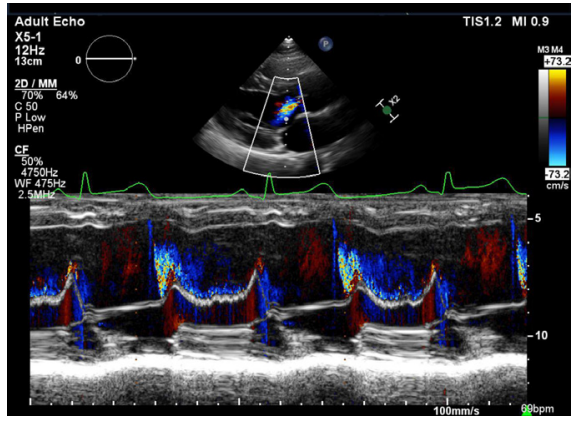

Figure 4 Colour M-mode echocardiographic image of the mitral valve. 
outcome requires that one caused, at least in part, that outcome but also that one had knowledge of the possibility and control over one's actions. Moral responsibility thus requires that an outcome is foreseeable and avoidable.' The differing models of no-responsibility, personal-responsibility or social responsibility are discussed, with model choice relevant to situations ranging from transplant organ allocation to missed clinic appointments.

The Image Challenge ${ }^{9}$ question is to identify the cause of a murmur based the image in figure 4.

Funding The authors have not declared a specific grant for this research from any funding agency in the public, commercial or not-for-profit sectors.

Competing interests None declared.
Patient and public involvement Patients and/or the public were not involved in the design, or conduct, or reporting, or dissemination plans of this research.

Patient consent for publication Not required.

Provenance and peer review Commissioned: internally peer reviewed.

(c) Author(s) (or their employer(s)) 2021. No commercial re-use. See rights and permissions. Published by BMJ.

\section{A) Check for updates}

To cite Otto CM. Heart 2021;107:685-687.

Heart 2021;107:685-687.

doi:10.1136/heartjnl-2021-319404

ORCID iD

Catherine M Otto http://orcid.org/0000-0002-05279392

\section{REFERENCES}

1 Rashid M, Timmis A, Kinnaird T, et al. Racial differences in management and outcomes of acute myocardial infarction during COVID-19 pandemic. Heart 2021;107:734-40.

2 Cader FA, Yancy CW, Banerjee S. To be, or not to be BAME, in the time of COVID-19: does it matter? Heart 2021;107:692-3.

3 Vasconcelos M, Vasconcelos L, Ribeiro V, et al. Incidence and predictors of stroke in patients with rheumatic heart disease. Heart 2021;107:748-54.

4 Karthikeyan G. Stroke risk in rheumatic heart disease. Heart 2021:107:694-6.

5 Hamatani Y, Iguchi M, Ueno K. Prognostic significance of natriuretic peptide levels in atrial fibrillation without heart failure. Heart 2021;107:705-12.

6 Guasch E. Natriuretic peptides in atrial fibrillation: evidence is here, but are we ready to go one step beyond? Heart 2021;107:688-9.

7 Mintz GS, Ali Z, Maehara A. Use of intracoronary imaging to guide optimal percutaneous coronary intervention procedures and outcomes. Heart 2021;107:755-64.

8 Brown RCH, Savulescu J. Personal responsibility for cardiac health: what are the ethical demands? Heart 2021;107:765-6.

9 Taylor AP, Hanson D, Krieger EV. Heart murmur in a young man. Heart 2021;107:770 